\title{
High IL-17-positive tumor immune cell infiltration is indicative for chemosensitivity of ovarian carcinoma
}

\author{
Raoul A. Droeser $\cdot$ Uwe Güth $\cdot$ Serenella Eppenberger-Castori • \\ Sylvia Stadlmann • Christian Hirt • Luigi Terracciano • \\ Gad Singer
}

Received: 11 January 2013/Accepted: 16 April 2013/Published online: 28 April 2013

(C) Springer-Verlag Berlin Heidelberg 2013

\begin{abstract}
Purpose Ovarian carcinoma in most instances is diagnosed in an advanced stage which cannot be cured by surgical tumor debulking alone. Standard adjuvant chemotherapy usually follows surgical procedures. Yet, few reliable predictive tissue markers exist for the response of ovarian carcinoma to chemotherapy. In this study, we evaluated the predictive value of IL-17- and FOXP3positive tumor immune cell infiltration (TICI) for response to chemotherapy in ovarian carcinoma.

Methods Formalin fixed and paraffin embedded biopsies of mostly high-grade primary serous ovarian carcinomas and their matched recurrences were immunostained with IL-17 and FOXP3 on a tissue microarray. Chemoresistance was defined as tumor recurrence within 6 months of the completion of platinum-based chemotherapy. In 94 and 90
\end{abstract}

\footnotetext{
R. A. Droeser $(\bowtie) \cdot$ C. Hirt

Department of Surgery, University Hospital Basel,

Spitalstrasse 21, 4031 Basel, Switzerland

e-mail: Raoul.Droeser@usb.ch

C. Hirt

e-mail: chirt@uhbs.ch

R. A. Droeser - C. Hirt

Institute for Surgical Research and Hospital Management ICFS,

Hebelstrasse 20, 4031 Basel, Switzerland

U. Güth

Department of Gynecology and Obstetrics, University Hospital

Basel, Spitalstrasse 21, 4031 Basel, Switzerland

e-mail: uwe.gueth@ksw.ch

U. Güth

Department of Gynecology and Obstetrics, Kantonsspital

Winterthur, Brauerstrasse 15, 8400 Winterthur, Switzerland
}

biopsies, conclusive data for IL-17 and FOXP3 were available, respectively.

Results IL-17, but not FOXP3-positive TICI, displayed a significantly higher density in biopsies of chemosensitive tumors $(p=0.01)$. No significant difference in the expression of IL-17 and FOXP3 TICI was observed in all paired primary and recurrent biopsies without respect to chemoresponse $(p=0.77$ and $p=0.87$, respectively). However, significantly more IL-17-positive recurrences were encountered in the group of patients with chemosensitive tumors $(p=0.008)$.

Conclusions High IL-17-positive TICI is indicative for response to chemotherapy in ovarian carcinoma. Higher frequency of IL-17-positive TICI might persist in recurrent tumor tissues of chemosensitive biopsies, suggesting repetitive platinum-based chemotherapy as an appropriate therapy for patients with IL-17-positive recurrences.

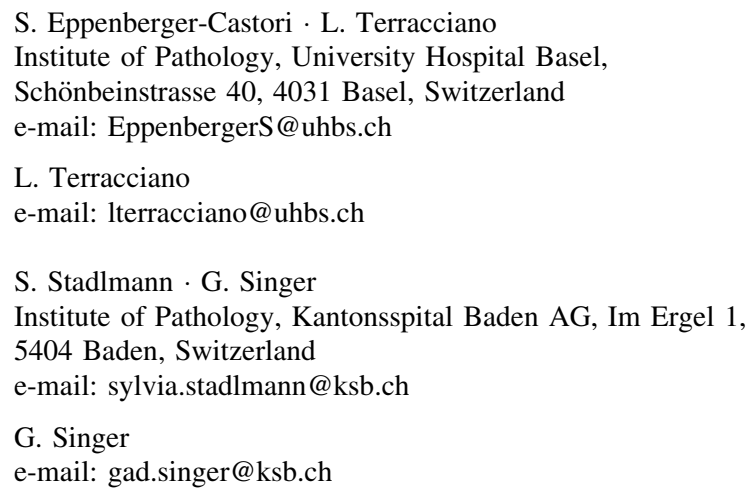


Keywords Forkhead box P3 - Tissue microarray . Regulatory T cell · Interleukin-17 · Chemosensitivity . Ovarian cancer

Abbreviations
$\begin{array}{ll}\text { FOXP3 } & \text { Forkhead box P3 } \\ \text { TMA } & \text { Tissue microarray } \\ \text { Treg } & \text { Regulatory T cell } \\ \text { IL-17 } & \text { Interleukin-17 }\end{array}$

\section{Introduction}

Ovarian carcinoma is the fifth most common female cancer with an incidence range of 5-15/100'000 in Europe, but the most letal of all female genital carcinomas (Jemal et al. 2010; Ferlay et al. 2010; Bray et al. 2005). Due to late and unspecific symptoms onset, it is in most instances diagnosed in advanced stages, which cannot be treated by surgery alone. Adjuvant platinum-based chemotherapy usually follows surgical debulking. The vast majority of patients recur with chemoresistant disease.

In order to optimize adjuvant therapy, earlier studies of ovarian cancer analyzed different markers predicting response to platinum-based chemotherapy, but few markers have been identified as useful ( $\mathrm{He}$ et al. 2008; Sato et al. 1999; Polcher et al. 2010). It is known that tumor biology influences tumor microenvironment and that tumor behavior is not only affected by the biologic behavior of the neoplastic cells. Evidence points to important influence of tumor-infiltrating immune cells on prognosis and tumor growth (Liotta and Kohn 2001; Stagg 2008; Uppaluri et al. 2008; Oble et al. 2009; Nosho et al. 2010). Tumor-infiltrating lymphocytes (TILs) are frequently considered to reflect host immune response to infiltrative neoplasms (Takagi et al. 1989). They have been shown to infiltrate a variety of tumors from different origins (Galon et al. 2006; $\mathrm{Su}$ et al. 2010). In ovarian cancer, it was shown that FOXP3 positive regulatory $\mathrm{T}$ (Treg) cell infiltration is associated with decreased survival (Wolf et al. 2005; Curiel et al. 2004; Polcher et al. 2010). Furthermore, it was shown that ovarian cancer-associated CD4-positive Treg cells can be converted into proinflammatory interleukin 17-producing helper T cells (Leveque et al. 2009). In addition, it is known that IL-17 can be produced by granulocytes ( $\mathrm{Li}$ et al. 2010; Lin et al. 2011) and other immune cells of the innate immune system (Cua and Tato 2010). The role of IL-17 tumor immune cell infiltration (TICI) in ovarian carcinoma is still debated. Kryczek et al. (2009) showed that high density of IL-17-positive TICI is associated with improved survival. The predictive value of IL-17- and FOXP3-positive TICI regarding chemotherapy in ovarian cancer therefore merits specific analysis. As the prognostic role of these two immune markers in ovarian carcinoma is well studied, we investigated their predictive value for chemosensitivity in a cohort of primary ovarian carcinomas and their matched recurrences (Stadlmann et al. 2006, 2007a, b, 2008).

\section{Materials and methods}

Patients

Tissues from ovarian serous carcinomas and their recurrences were collected from the Tissue Biobank Basel of the Institutes of Pathology of the University Hospital of Basel and the Cantonal Hospitals of Baden, Liestal and St. Gallen, Switzerland.

Mostly high-grade ovarian carcinomas (5.7\% FIGO stage II, $84.3 \%$ FIGO stage III and $4.3 \%$ FIGO stage IV) were included in this study after typing according to previous publications (Singer et al. 2002, 2003). A tissue microarray (TMA) was constructed which was also used in previous studies (Stadlmann et al. 2006, 2007a, b, 2008). All patients had recurrences after initial surgery and at least three cycles of platinum-based adjuvant chemotherapy. The collective was clinically divided into chemoresistant or chemosensitive tumors. Resistance was defined as recurrence occurring within 6 months after completion of platinum-based chemotherapy (Jazaeri et al. 2005). The TMA allowed investigation of tissues from ovarian carcinomas and matched recurrences from the same patients (Stadlmann et al. 2006, 2007a, b, 2008). The clinical data, including surgical and systemic treatment characteristics, were collected from medical records. All personal data were made anonymous. The present study was performed according to the guidelines of the institutional review boards of the participating institutions in compliance with specific regard to ethical standards and patient confidentiality. The use of this clinically annotated TMA for research was approved by the Regional Ethics Committee previously (Stadlmann et al. 2006, 2007a, b, 2008).

Tissue microarray construction

Tissue samples fixed in $4 \%$ buffered formalin and embedded in paraffin were used to construct a TMA (Sauter et al. 2003). Briefly, hematoxylin-eosin stained sections were made from each selected primary block (donor blocks) to define representative tissue regions. Tissue cylinders $(0.6 \mathrm{~mm}$ in diameter) were then punched from the region of the donor block with the use of a custom-made precision instrument (Beecher Instruments, Silver Spring, MD, USA). Tissue cylinders were transferred 
to a $25 \mathrm{~mm} \times 35 \mathrm{~mm}$ paraffin block to produce the TMA block used for the study. The resulting TMA block was cut into $3 \mu \mathrm{m}$ sections, which was transferred to glass slides by use of the Paraffin Sectioning Aid System (Instrumedics, Hackensacks, NJ, USA). Sections from the TMA block were used for the different analyses.

Immunohistochemistry (IHC) and analysis

Standard indirect immunoperoxidase procedures (ABCElite, Vectra Laboratories) were used for immunohistochemistry with the following antibodies: purified polyclonal goat anti-human IL-17 antibody (R\&D Systems, Minneapolis, MN, USA), dilution 1:50, polyclonal rabbit IL-17 antibody (sc-7927, H-132, Santa Cruz Biotechnology, Santa Cruz, CA, USA), dilution 1:10 and monoclonal mouse anti-human FOXP3 (clone 236A/E7, Abcam, Cambridge, UK), dilution 1:20. To generate the data used for comparison to chemosensitivity, the purified polyclonal goat anti-human IL-17 antibody (R\&D Systems, Minneapolis, MN, USA) was used.

Positively stained TICI in the stroma was counted in each tissue spot, representing approximately one highpower field $(400 \times)$, intravascular cells were excluded from analysis (Fig. 1b, d). Staining was evaluated for specificity and analyzed by two experienced observers (RD and GS). Cutoff was 1 positive cell/punch for both markers. In 94 and 90 biopsies, conclusive data for IL-17 and FOXP3 were available in primary and matched recurrent carcinomas, respectively.

\section{Validation analysis of IL-17 staining}

It is known that cells of the innate immune system can produce IL-17 (Cua and Tato 2010; Li et al. 2010; Lin et al. 2011). Even though that most of the IL-17-positive TICI resembled morphologically lymphocytes granulocytes seemed to stain for IL-17, too. Therefore, we performed a validation staining with two different IL-17 antibodies to assess specificity. The correlation between the two antibodies (AF-317-NA, R\&D systems and sc-7927 (H-132), Santa Cruz Biotechnology) was strong and highly significant $\left(r_{s}=0.78 ; p<0.0001\right)$.

The majority of all biopsies $(n=94)$ were found to be completely negative for IL-17 $(n=55)$. In the positive biopsies, the number of IL-17 protein expressing cells ranged from 1 to 93 .

\section{Statistical analysis section}

Cutoff scores used to classify ovarian carcinomas with low or high IL-17 or FOXP3 infiltration were obtained by regression tree analysis, evaluating sensitivity and false

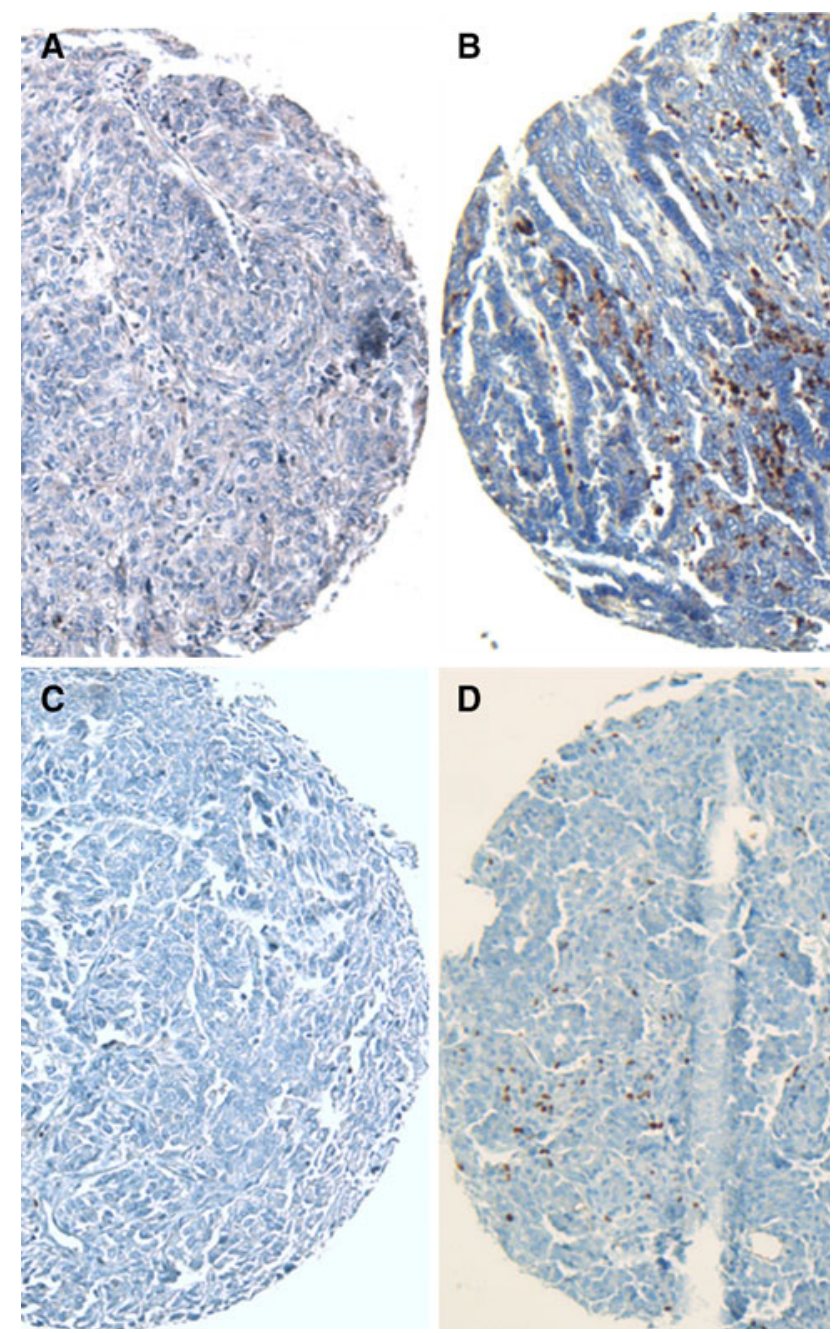

Fig. 1 IL-17- and FOXP3-specific staining in high-grade ovarian carcinoma. Tumor punches are representative of low (panels a and c) and high (panels $\mathbf{b}$ and d) density of IL-17-positive (panels $\mathbf{a}$ and b) and FOXP3-positive (panels $\mathbf{c}$ and d) TICI. Magnification: $\times 10$

positive rate for the discrimination of survivors and nonsurvivors, on all tumor samples (Zlobec et al. 2007). Specific scores were set at 1 positive cell/punch for both markers. Wilcoxon and Fisher's exact tests were used to determine the association of IL-17 and FOXP3 infiltration and clinicopathological features for continuous and categorical variables, respectively. Univariate recurrence-free and overall survival analyses were carried out by the Kaplan-Meier method and compared by means of log rank test.

The assumption of proportional hazards was verified for both markers by analyzing the correlation of Schoenfeld residuals and the ranks of individual failure times. Any missing clinicopathological information was assumed to be missing at random. Subsequently, IL-17 and FOXP3 cell infiltration data were entered into multivariate Cox regression analysis and hazard ratios (HR), and $95 \%$ confidence intervals (CI) were used to determine 
Table 1 Patients' characteristics according to dichotomized distribution of IL-17 and FOXP3 in the overall cohort

\begin{tabular}{|c|c|c|c|c|c|c|}
\hline & $\begin{array}{l}\text { IL-17 high } \\
N(\%)\end{array}$ & $\begin{array}{l}\text { IL-17 low } \\
N(\%)\end{array}$ & $p$ value & $\begin{array}{l}\text { FOXP3 high } \\
N(\%)\end{array}$ & $\begin{array}{l}\text { FOXP3 low } \\
N(\%)\end{array}$ & $p$ value \\
\hline Age (median, range) & $56(34-73)$ & $62(39-77)$ & 0.15 & $60(34-73)$ & $58(39-77)$ & 0.65 \\
\hline \multicolumn{7}{|l|}{ FIGO stage $^{a}$} \\
\hline I & $0(0)$ & $1(3.8)$ & 0.75 & $1(6.7)$ & $0(0)$ & 0.29 \\
\hline II & $2(10.5)$ & $1(3.8)$ & & $0(0)$ & $3(10.0)$ & \\
\hline III & $17(89.5)$ & $24(92.4)$ & & $14(93.3)$ & $27(90.0)$ & \\
\hline \multicolumn{7}{|l|}{ Resection status $^{\mathrm{a}}$} \\
\hline 0 & $6(30.0)$ & $10(38.5)$ & 0.84 & $4(26.7)$ & $11(36.7)$ & 0.79 \\
\hline 1 & $10(50.0)$ & 7 (26.9) & & $6(40.0)$ & $11(36.7)$ & \\
\hline 2 & $4(20.0)$ & $9(34.6)$ & & $5(33.3)$ & $8(26.6)$ & \\
\hline \multicolumn{7}{|l|}{ Chemotherapy $^{\mathrm{a}}$} \\
\hline$<6$ cycles & $2(8.7)$ & $5(21.7)$ & 0.44 & $2(13.3)$ & $5(16.7)$ & 0.99 \\
\hline 6 or more cycles & $21(91.3)$ & $18(78.3)$ & & $13(86.7)$ & $25(83.3)$ & \\
\hline RFS (mean/SE) ${ }^{\mathrm{b}}$ & $14.5 \pm 2.3$ & $6.4 \pm 1.4$ & 0.01 & $10.6 \pm 2.4$ & $9.9 \pm 1.7$ & 0.44 \\
\hline OS $(\text { mean/SE) })^{\mathrm{b}}$ & $55.7 \pm 9.0$ & $35.8 \pm 4.3$ & 0.05 & $44.6 \pm 10.0$ & $40.9 \pm 4.2$ & 0.59 \\
\hline
\end{tabular}

Missing clinicopathological information was assumed to be missing at random

${ }^{\text {a }}$ Fisher's exact test

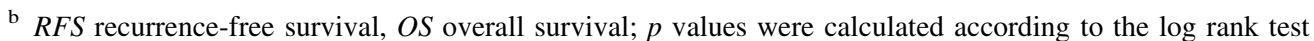

prognostic effects on survival time. Spearman's rank correlation was used to analyze the correlation between the two different IL-17 antibodies and between IL-17 and FOXP3. Statistical analyses were performed using SPlus software (Version 6.1, Insightful Corporation, Seattle, WA, USA) and SAS (V9.1, the SAS Institute, Cary, NC).

\section{Results}

Patient characteristics

Patient's median age was 58.5 (34-77) in the chemosensitive and 63 (27-77) in the chemoresistant group, respectively. Recurrence-free and overall survival in the chemoresistant group were significantly shorter than in the chemosensitive group $(2.2 \pm 0.3$ vs. $18.2 \pm 2.0$ months, $p<0.0001$ and $27 \pm 5.3$ vs. $49.6 \pm 4.0$ months, $p=0.0003$, respectively). Most patients $(94.3 \%)$ had histopathologically high-grade serous papillary carcinomas, and the two groups were comparable according to the FIGO classification $(p=0.47)$. Furthermore, the analysis of clinicopathological data of the overall cohort by density of IL-17- and FOXP3-positive TICI is summarized in Table 1.

IL-17-positive immune cell infiltration in paired primary and recurrent ovarian carcinoma

Only 2 out of $14(14.3 \%)$ chemoresistant primary carcinomas were classified as positive for $\mathrm{IL}-17^{+}$immune cell infiltration. In the chemosensitive subset, 18 out of 33
$(54.5 \%)$ primary carcinomas were positive for IL-17 cell infiltration (Table 2). Similarly, 2 out of 14 (14.3\%) recurrent chemoresistant carcinomas and 17 out of $33(51.5 \%)$ recurrent chemosensitive carcinomas were positive for IL-17, respectively. Ten IL-17-negative primary carcinomas samples were positive in their corresponding recurrences. Remarkably, only 2 of them belonged to the chemoresistant patients subset. Nine samples from the chemosensitive patients subset retained their positivity for IL-17 TICI in the corresponding recurrent carcinomas $(p=0.008)$.

FOXP3-positive immune cell infiltration in paired primary and recurrent ovarian carcinoma

Six out of $13(46.2 \%)$ primary carcinomas of the chemoresistant and 9 out of $32(28.1 \%)$ of the chemosensitive subset patients were positive for FOXP3 cell infiltration. The paired recurrences showed 4 out of $13(30.8 \%)$ chemoresistant carcinomas and 10 out of $32(31.2 \%)$ chemosensitive carcinomas to be positive for FOXP3. Seven patients that were negative in their primary carcinomas turned to be positive for FOXP3 in their recurrent tumors. Of these 7 patients, 2 were from the chemoresistant subset. Another 7 patients were positive for FOXP3 infiltration in the primary and recurrent carcinoma samples ( 2 chemoresistant; 5 chemosensitive; Table 2).

Distribution of IL-17- and FOXP3-positive TICI according to chemosensitivity

As shown in Fig. 2, IL-17-positive TICI frequency was significantly higher in the chemosensitive group in all 
Table 2 Dichotomized distribution of IL-17 and FOXP3 according to defined cutoff $(1 \mathrm{cell} / \mathrm{punch})$ in primary and matched recurrent carcinomas

\begin{tabular}{llll}
\hline $\begin{array}{l}\text { Chemoresistant } \\
\text { tumors } \\
n=14(100 \%)\end{array}$ & $\begin{array}{l}\text { Chemosensitive } \\
\text { tumors } \\
n=33(100 \%)\end{array}$ & $p$ value \\
\hline
\end{tabular}

\begin{tabular}{lccc}
\hline$I L-17$ & & & \\
Primary & & & \\
Positive & $2(14.3)$ & $18(54.5)$ & 0.02 \\
Negative & $12(85.7)$ & $15(45.5)$ & \\
Recurrent & & & 0.02 \\
Positive & $2(14.3)$ & $16(51.5)$ & \\
Negative & $12(85.7)$ & $n=32(100 \%)$ & \\
\hline & $n=13(100 \%)$ & & 0.3 \\
\hline FOXP3 & & $9(28.1)$ & \\
Primary & & $23(71.9)$ & \\
Positive & $6(46.2)$ & $10(31.2)$ & \\
Negative & $7(53.8)$ & $22(68.8)$ & \\
Recurrent & & & \\
Positive & $4(30.8)$ & & \\
Negative & $9(69.2)$ & &
\end{tabular}

biopsies $(n=94, p=0.001)$ as well as in biopsies of primary $(n=47, p=0.01)$ and matched recurrent $(n=47, p=0.04)$ carcinomas. For FOXP3-positive cell infiltration, no association with sensitivity to platinumbased chemotherapy could be determined in any of the three groups $(p=0.53, p=0.35$ and $p=0.91$, respectively; Fig. 2).

Prognostic significance of IL-17- and FOXP3-positive tumor immune cell infiltration

In the overall cohort of primary and matched recurrent ovarian carcinomas, IL-17 $(n=47)$ had a positive prognostic effect in univariate analysis $(p=0.045)$. However, both markers were devoid of any prognostic effect in multivariate analysis.

Correlation analysis of IL-17- and FOXP3-positive tumor immune cell infiltration

To obtain more information about the relationship of the two different subsets of immune cells, a correlation analysis of IL17- and FOXP3-positive cells was performed. However, IL-17- and FOXP3-positive TICI did neither correlate significantly in all biopsies $\left(r_{\mathrm{s}}=0.17 ; p=0.1\right)$ nor in biopsies of only primary $\left(r_{\mathrm{s}}=0.05 ; p=0.73\right)$ or recurrent carcinomas $\left(r_{\mathrm{s}}=0.29 ; p=0.054\right)$.

\section{Discussion}

Adjuvant platinum-based chemotherapy after surgical tumor debulking is a standard procedure. It would be helpful to find markers that help predict response to chemotherapy, in order to include extended chemotherapy regimen and repetitive surgical procedures. Although our previous studies identified potential therapeutical targets in chemoresistant ovarian carcinomas, a reliable predictive marker was not detected (Stadlmann et al. 2006, 2007a, b, 2008).

In this study, we found that IL-17-positive TICI was significantly more frequent in the chemosensitive ovarian carcinoma group. The presence of IL-17 positivity was found to be very similar in primary and recurrent tissues. IL-17-positive TICI was retained in $45 \%(9 / 20)$ of recurrent carcinomas. Even though that in another $45 \%$ (21/47), the IL-17 status between primary and recurrent tumors switched from positive to negative or vice versa IL-17positive biopsies were still increased in numbers in the chemosensitive group. Therefore, IL-17 may keep its indicative value for response to platinum-based chemotherapy in recurrent carcinomas. On the other hand, these tumors might simply be less aggressive, either due to host immune or other factors.

FOXP3 is a transcription factor that is mainly expressed in so called regulatory cells with immunosuppressive function (Zou 2006; Tang and Bluestone 2008). In ovarian cancer, FOXP3 positive Treg cell infiltration has been shown to be associated with significantly worse survival. In this study, FOXP3-positive TICI had no predictive value for response to chemotherapy.

Reviewing the literature, there is an ongoing search for reliable prognostic and predictive markers in ovarian cancer. There is a trend toward individualized treatment based on chemosensitivity assays or gene microarray analyses (Kigawa et al. 2001; Sato et al. 1999). Tumor immune response is known to play a major role in the control of tumor progression in different types of cancer. Tumor infiltration by $\mathrm{CD} 8$-positive $\mathrm{T}$ cells has been shown to represent an important prognostic factor in melanoma (Oble et al. 2009) and, more recently, in colorectal cancers (Galon et al. 2006; Pages et al. 2005). Early studies have also suggested a favorable prognostic effect of lymphocyte infiltration in breast cancers (Menard et al. 1997). More recently, tumor infiltration by $\mathrm{CD} 3$-positive $\mathrm{T}$ cells has been proposed to predict responsiveness to neoadjuvant treatment in breast cancer (Denkert et al. 2010). Furthermore, a predictive effect of breast cancer infiltration by FOXP3-positive cells has also been reported (de Kruijf et al. 2010). More aggressive tumors are growing faster and therefore may produce stromal damage possibly related to local hypoxia with necrotic areas (Minervini et al. 2008; 


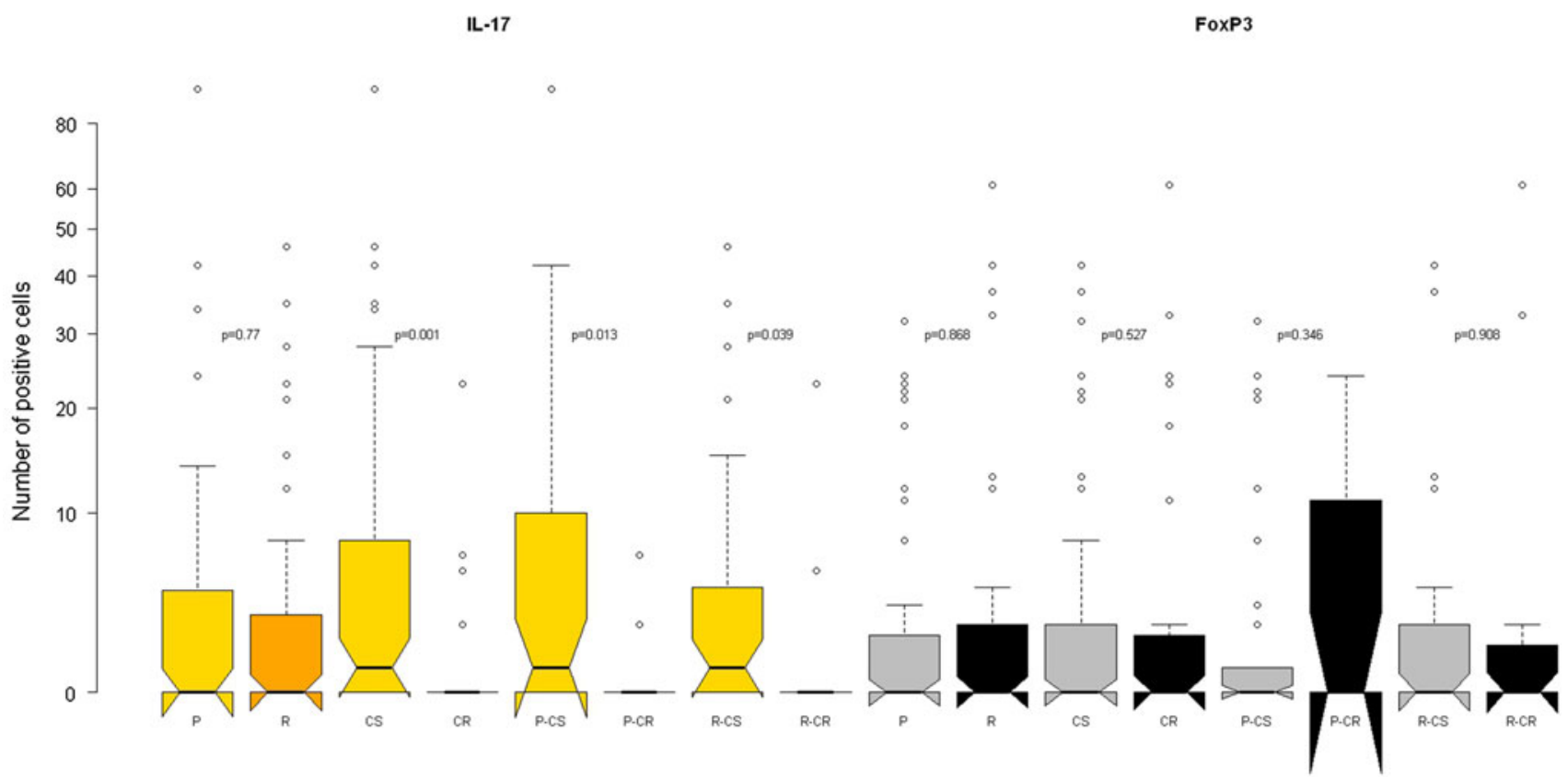

Fig. 2 Boxplot distribution of IL-17 and FOXP3 TICI in ovarian carcinomas overall and with respect to chemosensitivity. Boxplot distribution of IL-17 and FOXP3-positive immune cell infiltration in primary $(\mathrm{P})$ and matched recurrent $(\mathrm{R})$ carcinomas in the overall

Osinsky et al. 2009; De Schutter et al. 2005; Ben Shoshan et al. 2008). Increased numbers of CD4- and FOXP3positive cells under hypoxic condition could be shown in several studies (Ben Shoshan et al. 2008). Altogether the pattern of immune cell infiltration in solid tumors most likely reflects the state of tumor microenvironment and consequently might predict response to chemotherapy in ovarian cancer (Polcher et al. 2010).

Th17 cells, a subset of $\mathrm{CD}^{+}{ }^{+} \mathrm{T}$ cells characterized by the secretion of high levels of IL-17A, IL-17F and IL-22, play an important role in both the immune response to invading pathogens and autoimmunity as well as transplant rejection (Bettelli et al. 2008). The specific role of IL-17positive TICI in ovarian cancer is still debated. There is increasing evidence that IL-17 can be produced by granulocytes ( $\mathrm{Li}$ et al. 2010; Lin et al. 2011) and other immune cells of the innate immune system (Cua and Tato 2010). In ovarian cancer, it was shown that CD4-positive Treg cells could be converted into proinflammatory interleukin 17-producing helper T cells (Leveque et al. 2009). Kryczek et al. (2009) showed that a high density of IL-17-positive TICI is associated with better survival. On the other hand, steady production of TNF-alpha in the tumor microenvironment could increase myeloid cell recruitment in an IL17-dependent manner and contribute to tumor promotion (Charles et al. 2009).

The main limitations of this study are a possible sampling error, but pooling of primary and recurrent samples $(n=94$ patient cohort and with respect to chemoresponse in all biopsies (CS; $\mathrm{CR}$ ), primary (P-CS; P-CR) and matched recurrent (R-CS; R-CR) carcinomas; $C S$ chemosensitive, $C R$ chemoresistant

and $n=90$, respectively) confirmed that IL-17, but not FOXP3-positive TICI was significantly more frequent in biopsies of chemosensitive than in biopsies of chemoresistant tumors (Fig. 2). The potential problem of sufficient sampling is negligible regarding the statistical significance of our results since the low cut off level of one single positive cell/core was applied to all cases and all cores.

In conclusion, IL-17-positive immune cell infiltration could be a promising predictive marker in ovarian carcinoma. It remains to be confirmed in larger series. Furthermore, it would be interesting to investigate if IL-17 might also be of predictive value for second-line (platinumbased) chemotherapy in ovarian carcinoma.

Acknowledgments This study was funded by the Swiss Cancer League (Oncosuisse), Grant number OCS 01506-02-2004 for G.S.

Conflict of interest The authors declare that they have no financial competing interests.

\section{References}

Ben Shoshan J, Maysel-Auslender S, Mor A, Keren G, George J (2008) Hypoxia controls CD4+ CD25+ regulatory T-cell homeostasis via hypoxia-inducible factor-1alpha. Eur J Immunol 38:2412-2418

Bettelli E, Korn T, Oukka M, Kuchroo VK (2008) Induction and effector functions of $\mathrm{T}(\mathrm{H}) 17$ cells. Nature 453:1051-1057 
Bray F, Loos AH, Tognazzo S, La VC (2005) Ovarian cancer in Europe: cross-sectional trends in incidence and mortality in 28 countries, 1953-2000. Int J Cancer 113:977-990

Charles KA, Kulbe H, Soper R, Escorcio-Correia M, Lawrence T, Schultheis A, Chakravarty P, Thompson RG, Kollias G, Smyth JF, Balkwill FR, Hagemann T (2009) The tumor-promoting actions of TNF-alpha involve TNFR1 and IL-17 in ovarian cancer in mice and humans. J Clin Invest 119:3011-3023

Cua DJ, Tato CM (2010) Innate IL-17-producing cells: the sentinels of the immune system. Nat Rev Immunol 10:479-489

Curiel TJ, Coukos G, Zou L, Alvarez X, Cheng P, Mottram P, Evdemon-Hogan M, Conejo-Garcia JR, Zhang L, Burow M, Zhu Y, Wei S, Kryczek I, Daniel B, Gordon A, Myers L, Lackner A, Disis ML, Knutson KL, Chen L, Zou W (2004) Specific recruitment of regulatory $\mathrm{T}$ cells in ovarian carcinoma fosters immune privilege and predicts reduced survival. Nat Med 10:942-949

de Kruijf EM, van Nes JG, Sajet A, Tummers QR, Putter H, Osanto S, Speetjens FM, Smit VT, Liefers GJ, van de Velde CJ, Kuppen PJ (2010) The predictive value of HLA class I tumor cell expression and presence of intratumoral Tregs for chemotherapy in patients with early breast cancer. Clin Cancer Res 16:1272-1280

De Schutter H, Landuyt W, Verbeken E, Goethals L, Hermans R, Nuyts S (2005) The prognostic value of the hypoxia markers CA IX and GLUT 1 and the cytokines VEGF and IL 6 in head and neck squamous cell carcinoma treated by radiotherapy \pm chemotherapy. BMC Cancer 5:42

Denkert C, Loibl S, Noske A, Roller M, Muller BM, Komor M, Budczies J, Darb-Esfahani S, Kronenwett R, Hanusch C, von Torne C, Weichert W, Engels K, Solbach C, Schrader I, Dietel M, von Minckwitz G (2010) Tumor-associated lymphocytes as an independent predictor of response to neoadjuvant chemotherapy in breast cancer. J Clin Oncol 28:105-113

Ferlay J, Parkin DM, Steliarova-Foucher E (2010) Estimates of cancer incidence and mortality in Europe in 2008. Eur J Cancer 46:765-781

Galon J, Costes A, Sanchez-Cabo F, Kirilovsky A, Mlecnik B, Lagorce-Pages C, Tosolini M, Camus M, Berger A, Wind P, Zinzindohoue $\mathrm{F}$, Bruneval $\mathrm{P}$, Cugnenc $\mathrm{PH}$, Trajanoski $\mathrm{Z}$, Fridman WH, Pages F (2006) Type, density, and location of immune cells within human colorectal tumors predict clinical outcome. Science 313:1960-1964

He Z, Gao J, Wang Q, Liu M, Li Y, Li X, Tang H, Zheng J (2008) S100P contributes to chemosensitivity of human ovarian cancer cell line OVCAR3. Oncol Rep 20:325-332

Jazaeri AA, Awtrey CS, Chandramouli GV, Chuang YE, Khan J, Sotiriou C, Aprelikova O, Yee CJ, Zorn KK, Birrer MJ, Barrett JC, Boyd J (2005) Gene expression profiles associated with response to chemotherapy in epithelial ovarian cancers. Clin Cancer Res 11:6300-6310

Jemal A, Siegel R, Xu J, Ward E (2010) Cancer statistics, 2010. CA Cancer J Clin 60:277-300

Kigawa J, Sato S, Shimada M, Takahashi M, Itamochi H, Kanamori Y, Terakawa N (2001) p53 gene status and chemosensitivity in ovarian cancer. Hum Cell 14:165-171

Kryczek I, Banerjee M, Cheng P, Vatan L, Szeliga W, Wei S, Huang E, Finlayson E, Simeone D, Welling TH, Chang A, Coukos G, Liu R, Zou W (2009) Phenotype, distribution, generation, and functional and clinical relevance of Th17 cells in the human tumor environments. Blood 114:1141-1149

Leveque L, Deknuydt F, Bioley G, Old LJ, Matsuzaki J, Odunsi K, Ayyoub M, Valmori D (2009) Interleukin 2-mediated conversion of ovarian cancer-associated $\mathrm{CD} 4+$ regulatory $\mathrm{T}$ cells into proinflammatory interleukin 17-producing helper T cells. J Immunother 32:101-108
Li L, Huang L, Vergis AL, Ye H, Bajwa A, Narayan V, Strieter RM, Rosin DL, Okusa MD (2010) IL-17 produced by neutrophils regulates IFN-gamma-mediated neutrophil migration in mouse kidney ischemia-reperfusion injury. J Clin Invest 120:331-342. doi:10.1172/JCI38702

Lin AM, Rubin CJ, Khandpur R, Wang JY, Riblett M, Yalavarthi S, Villanueva EC, Shah P, Kaplan MJ, Bruce AT (2011) Mast cells and neutrophils release IL-17 through extracellular trap formation in psoriasis. J Immunol 187:90-500. doi:10.4049/jimmunol.1 100123

Liotta LA, Kohn EC (2001) The microenvironment of the tumourhost interface. Nature 411:375-379

Menard S, Tomasic G, Casalini P, Balsari A, Pilotti S, Cascinelli N, Salvadori B, Colnaghi MI, Rilke F (1997) Lymphoid infiltration as a prognostic variable for early-onset breast carcinomas. Clin Cancer Res 3:817-819

Minervini A, Di Cristofano C, Gacci M, Serni S, Menicagli M, Lanciotti M, Salinitri G, Rocca CD, Lapini A, Nesi G, Bevilacqua G, Minervini R, Carini M (2008) Prognostic role of histological necrosis for nonmetastatic clear cell renal cell carcinoma: correlation with pathological features and molecular markers. J Urol 180:1284-1289

Nosho K, Baba Y, Tanaka N, Shima K, Hayashi M, Meyerhardt JA, Giovannucci E, Dranoff G, Fuchs CS, Ogino S (2010) Tumourinfiltrating T-cell subsets, molecular changes in colorectal cancer, and prognosis: cohort study and literature review. J Pathol 222:350-366

Oble DA, Loewe R, Yu P, Mihm MC Jr (2009) Focus on TILs: prognostic significance of tumor infiltrating lymphocytes in human melanoma. Cancer Immun 9:3

Osinsky S, Zavelevich M, Vaupel P (2009) Tumor hypoxia and malignant progression. Exp Oncol 31:80-86

Pages F, Berger A, Camus M, Sanchez-Cabo F, Costes A, Molidor R, Mlecnik B, Kirilovsky A, Nilsson M, Damotte D, Meatchi T, Bruneval P, Cugnenc PH, Trajanoski Z, Fridman WH, Galon J (2005) Effector memory T cells, early metastasis, and survival in colorectal cancer. N Engl J Med 353:2654-2666

Polcher M, Braun M, Friedrichs N, Rudlowski C, Bercht E, Fimmers R, Sauerwald A, Keyver-Paik MD, Kubler K, Buttner R, Kuhn WC, Hernando JJ (2010) Foxp3(+) cell infiltration and granzyme $\mathrm{B}(+) /$ Foxp3(+) cell ratio are associated with outcome in neoadjuvant chemotherapy-treated ovarian carcinoma. Cancer Immunol Immunother 59:909-919

Sato S, Kigawa J, Minagawa Y, Okada M, Shimada M, Takahashi M, Kamazawa S, Terakawa N (1999) Chemosensitivity and p53dependent apoptosis in epithelial ovarian carcinoma. Cancer 86:1307-1313

Sauter G, Simon R, Hillan K (2003) Tissue microarrays in drug discovery. Nat Rev Drug Discov 2:962-972

Singer G, Kurman RJ, Chang HW, Cho SK, Shih I (2002) Diverse tumorigenic pathways in ovarian serous carcinoma. Am J Pathol 160:1223-1228

Singer G, Oldt R III, Cohen Y, Wang BG, Sidransky D, Kurman RJ, Shih I (2003) Mutations in BRAF and KRAS characterize the development of low-grade ovarian serous carcinoma. J Natl Cancer Inst 95:484-486

Stadlmann S, Gueth U, Reiser U, Diener PA, Zeimet AG, Wight E, Mirlacher M, Sauter G, Mihatsch MJ, Singer G (2006) Epithelial growth factor receptor status in primary and recurrent ovarian cancer. Mod Pathol 19:607-610

Stadlmann S, Gueth U, Baumhoer D, Moch H, Terracciano L, Singer G (2007a) Glypican-3 expression in primary and recurrent ovarian carcinomas. Int J Gynecol Pathol 26:341-344

Stadlmann S, Gueth U, Wight E, Kunz-Schughart LA, Hartmann A, Singer G (2007b) Expression of peroxisome proliferator 
activated receptor gamma and cyclo-oxygenase 2 in primary and recurrent ovarian carcinoma. J Clin Pathol 60:307-310

Stadlmann S, Dirnhofer S, Guth U, Thies S, Singer G (2008) ERCC1immunoexpression does not predict platinum-resistance in ovarian cancer. Gynecol Oncol 108:252-253

Stagg J (2008) Mesenchymal stem cells in cancer. Stem Cell Rev 4:119-124

Su X, Ye J, Hsueh EC, Zhang Y, Hoft DF, Peng G (2010) Tumor microenvironments direct the recruitment and expansion of human Th17 cells. J Immunol 184:1630-1641

Takagi S, Chen K, Schwarz R, Iwatsuki S, Herberman RB, Whiteside TL (1989) Functional and phenotypic analysis of tumorinfiltrating lymphocytes isolated from human primary and metastatic liver tumors and cultured in recombinant interleukin-2. Cancer 63:102-111

Tang Q, Bluestone JA (2008) The Foxp3+ regulatory T cell: a jack of all trades, master of regulation. Nat Immunol 9:239-244
Uppaluri R, Dunn GP, Lewis JS Jr (2008) Focus on TILs: prognostic significance of tumor infiltrating lymphocytes in head and neck cancers. Cancer Immun 8:16

Wolf D, Wolf AM, Rumpold H, Fiegl H, Zeimet AG, Muller-Holzner E, Deibl M, Gastl G, Gunsilius E, Marth C (2005) The expression of the regulatory $\mathrm{T}$ cell-specific forkhead box transcription factor FoxP3 is associated with poor prognosis in ovarian cancer. Clin Cancer Res 11:8326-8331

Zlobec I, Steele R, Terracciano L, Jass JR, Lugli A (2007) Selecting immunohistochemical cut-off scores for novel biomarkers of progression and survival in colorectal cancer. J Clin Pathol 60:1112-1116. doi:10.1136/jcp.2006.044537

Zou W (2006) Regulatory T cells, tumour immunity and immunotherapy. Nat Rev Immunol 6:295-307 